'Departamento de Psiquiatría, Escuela de Medicina, Pontificia Universidad Católica de Chile. Santiago, Chile.

${ }^{2}$ Escuela de Psicología, Pontificia Universidad Católica de Chile. Santiago, Chile.

'Departamento de Salud Pública, Escuela de Medicina, Pontificia Universidad Católica de Chile. Santiago, Chile. apsicólogo/a. bEstadístico.

Investigación financiada por proyecto FONDECYT (\#1030431). La organización que financió el proyecto no tuvo influencia en el diseño, ni en la ejecución del estudio, como tampoco en la preparación de este manuscrito.

Recibido el 12 de agosto de 2015, aceptado el 19 de enero de 2016.

Correspondencia a:

Dr. Rodrigo Santis Av. Camino El Alba 12351, Las Condes. Santiago, Chile Código Postal 7620002 Teléfono: (562) 27548872 Fax: (562) 27548878 rsantis@med.puc.c

\section{Intentos de suicidio y heridas auto-inferidas: 12 meses de seguimiento de conductas de riesgo de consumidores de cocaína no consultantes de servicios de rehabilitación}

\author{
RODRIGO SANTIS ${ }^{1}$, CARMEN GLORIA HIDALGO ${ }^{2, a}$, \\ VIVIANA HAYDEN ${ }^{1, \mathrm{a}}$, ENZO ANSELMO ${ }^{1, \mathrm{a}}$, ANDREA JARAMILLO ${ }^{2, \mathrm{a}}$, \\ OSLANDO PADILLA ${ }^{3, \mathrm{~b}}$, RAFAEL TORRES ${ }^{1}$
}

\section{Suicide attempts and self inflicted harm:} a one year follow up of risk behaviors among out of treatment cocaine users

Background: Cocaine users tend to have a higher frequency of risk behaviors. Aim: To compare risk behaviors of out of treatment Cocaine Base Paste (CBP) and Cocaine Hydrochloride $(\mathrm{CH})$ users, by means of Privileged Access Interviewing in a one year prospective study. Material and Methods: Twenty eight interviewers were trained to recruit and administer a questionnaire on substance use patterns and related risk behaviors. Intentioned sampling was carried out in four municipalities of Santiago, Chile. Subjects who used CBP (group 1) or $\mathrm{CH}$ (group 2) at least once in the last month, with primary current use of CBP (group 1) or $\mathrm{CH}$ (group 2), and without treatment for substance abuse in the last six months were interviewed. Generalized Estimating Equations (GEE) were employed to compare risk behaviors during follow up. Results: Four hundred and two of 467 subjects (86.1\%) were followed up for one year. CBP users $(n=204)$ reported greater frequency of self inflicted injuries (Odds Ratio (OR): 1.97 [95\% confidence intervals (CI): 1.073.66]) and suicide attempts (OR: 2.68 [95\% CI: 1.196.01]) than CH users. Conclusions: CBP users had a greater frequency of self inflicted injuries and suicide attempts, both life threatening risk behaviors, than $\mathrm{CH}$ users. This profile shows the high vulnerability of CBP users and should encourage further research and design of outreach interventions, particularly focused on this group.

(Rev Med Chile 2016; 144: 526-533)

Key words: Cocaine, Cocaine-Related Disorders, Dangerous Behavior.

\footnotetext{
C
} onocer el perfil de conductas de riesgo asociado a trastornos por consumo de sustancias específicas es epidemiológica y clínicamente relevante, tanto para diseñar estrategias preventivas focalizadas, como también intervenciones de tratamiento ajustadas a los problemas que afectan a los consumidores de sustancias.

El consumo de pasta base de cocaína (PBC), una sustancia intermedia en la producción de clorhidrato de cocaína (CC $)^{1,2}$, ha sido reportado en las últimas tres décadas en la región andina de Sudamérica ${ }^{1,3,4}$. Chile, Perú y Uruguay, tienen las prevalencias más altas de consumo de PBC en la última década ${ }^{5}$. Las prevalencias nacionales de consumo de último año son $1,4 \%$ para CC y $0,5 \%$ para $\mathrm{PBC}^{6}$. 
El consumo de cocaína afecta predominantemente a poblaciones vulnerables socioeconómicamente, y es la segunda sustancia ilegal más consumida en Chile después de marihuana ${ }^{6}$. Encuestas nacionales de población general muestran que la $\mathrm{PBC}$ es la sustancia ilegal que genera mayor dependencia, alcanzando $65,6 \%$ entre consumidores de último año $0^{6}$. Sin embargo, solamente $6,1 \%$ de los dependientes de sustancias ilegales, y $17,8 \%$ de los dependientes de PBC ingresa a tratamiento de rehabilitación cada $\mathrm{año}^{4}$, cifras comparables a lo reportado a nivel mundial: uno de cada 6 consumidores problemáticos o dependientes de drogas recibe tratamiento cada año ${ }^{7}$. Las conductas socialmente sancionadas, que suelen acompañar al consumo, son barreras para el contacto con los servicios de salud. Este grupo de consumidores, marginados de los sistemas de apoyo social, han sido denominados "poblaciones ocultas" $", 9$. La entrevista de acceso privilegiado (EAP) es una metodología que permite el estudio de estas poblaciones ${ }^{10,11}$, permitiendo reunir información a través de una red de entrevistadores que tienen acceso a los sujetos de estudio. Santis y cols. en $2006^{12}$, mediante la EAP, reportaron un alto grado de vulnerabilidad social y conductual de los consumidores de PBC no consultantes.

La primera publicación de la presente investigación presentó los datos de la evaluación basal ${ }^{13}$. Se mostró que los consumidores de PBC no consultantes reportaron una mayor proporción de conductas de riesgo en comparación a los consumidores de CC no consultantes. Adicionalmente, los consumidores de PBC provenían de familias más disfuncionales que los consumidores de $\mathrm{CC}^{14}$.

El objetivo del presente reporte es comparar el perfil de conductas de riesgo de una muestra de consumidores de PBC y consumidores de CC luego de 12 meses de seguimiento, sin tratamiento de rehabilitación en los 6 meses previos al inicio del estudio.

\section{Método}

La descripción detallada de participantes y método se encuentra publicada en reportes pre$\operatorname{vios}^{13,15}$. Se efectuó un diseño prospectivo con tres evaluaciones: ingreso, 6 meses y 12 meses de seguimiento. La metodología "EAP" fue utilizada para reclutar a la muestra ${ }^{10,11}$. Se seleccionaron y entrenaron 28 entrevistadores: 18 mujeres y 10 hombres. El reclutamiento, y las evaluaciones posteriores, se efectuaron en calles, plazas y espacios públicos de zonas de riesgo, definidas por mapas confeccionados con información de organizaciones locales y de Servicio Nacional para la Prevención y Rehabilitación del Consumo de Drogas y Alcohol (SENDA) respecto de áreas de alto consumo y tráfico de drogas, en cuatro municipios de la Región Metropolitana de Santiago de Chile, con las más alta prevalencias de consumo de $\mathrm{PBC}$ y CC, según el último estudio nacional de representación comunal $^{16}$ : Pedro Aguirre Cerda, Huechuraba, La Pintana y San Joaquín. El muestreo fue intencionado (no probabilístico) y de acuerdo al criterio de búsqueda de los entrevistadores dentro de cada zona de riesgo. La muestra fue estratificada de acuerdo al género (2/3 masculino) y rango etario (menos de 19 años, entre 19 y 25 años, y mayor de 25 años; un tercio cada categoría). Los criterios de inclusión fueron:

Grupo 1: Sujetos que consumieron PBC, como sustancia de primera elección, al menos una vez durante el último mes.

Grupo 2: Sujetos que consumieron CC, como sustancia de primera elección, al menos una vez durante el último mes.

Ambos grupos incluyeron sujetos sin tratamiento de rehabilitación en los 6 meses previos al inicio del estudio. Aquellos que no pudieron definir su sustancia primaria entre PBC y CC fueron excluidos.

La muestra consistió de 467 sujetos: 231 consumidores primarios de PBC (grupo 1) y 236 consumidores primarios de CC (grupo 2).

Los entrevistadores recibieron un pago de $\$ 6.000$ (US\$ 11), \$ 9.000 (US\$17) y \$ 11.000 (US\$20) por cada "Cuestionario de Conductas de Riesgo (CCR)" correctamente aplicado, al inicio, 6 y 12 meses de seguimiento, respectivamente. Los sujetos de estudio no recibieron pago por la evaluación inicial, pero sí recibieron cupones de $\$ 5.000$ (US\$ 9) y 8.000 (US\$15), canjeables por alimentos en un supermercado, a los 6 y 12 meses de seguimiento, respectivamente.

El instrumento empleado fue el $\mathrm{CCR}^{13}$ que evalúa: información sociodemográfica; patrón de consumo y dependencia de sustancias ( 3 o más de 6 criterios de acuerdo a CIE-10 $)^{17}$; severidad de la dependencia (número de criterios de dependencia de acuerdo a CIE-10 [mínimo: 3-máximo: 6]); policonsumo (consumo de dos o más sustancias en el 
último mes); polidependencia (presencia de dos o más dependencias, excluyendo tabaco); conductas de riesgo (prevalencia de vida y de últimos 6 meses de sobredosis, heridas auto-inferidas, intentos de suicidio, conductas sexuales de riesgo (no usar métodos anticonceptivos, pagar o recibir dinero a cambio de sexo, dar o recibir drogas a cambio de sexo), negligencia en el cuidado de menores, comisión de delitos (tráfico de drogas, robo en tiendas o supermercados, estafa, robo en casas o autos, asalto a personas, prostitución y porte de armas de fuego o armas corto-punzantes); estado y procesos legales; y atención sanitaria en servicios de salud. El análisis de confiabilidad y consistencia interna del CCR se detalla en reporte previo ${ }^{13}$.

El análisis estadístico se realizó mediante test $\mathrm{t}$ de Student, test de ANOVA de mediciones repetidas, $\chi^{2}$ y el cálculo de Odd Ratios (OR) mediante ecuaciones de estimación generalizada. Se consideró significativo un error máximo de 5\%. Las OR fueron ajustadas de acuerdo a potenciales factores confundentes: situación laboral, nivel educacional, deserción escolar, disfunción familiar ${ }^{14}$ y síntomas emocionales medidos por el cuestionario de salud general de Goldberg de 12 ítemes ${ }^{18}$.

Esta investigación fue aprobada por el Comité de Ética de la Escuela de Medicina de la Pontificia Universidad Católica de Chile.

\section{Resultados}

\section{Características socio-demográficas}

Ambos grupos estuvieron constituidos por participantes predominantemente solteros y casi $70 \%$ vivía con su familia de origen. El grupo 1 tuvo menor nivel educacional, mayor deserción escolar, mayor cesantía y empleos informales, que el grupo 2. La Tabla 1 presenta las características sociodemográficas basales de ambos grupos.

Tabla 1. Variables sociodemográficas de consumidores de pasta base de cocaína (Grupo 1 = 231 sujetos) y consumidores de clorhidrato de cocaína (Grupo 2= 236 sujetos) no consultantes al inicio del seguimiento

\begin{tabular}{|c|c|c|c|c|}
\hline & \multicolumn{2}{|c|}{ Grupo 1} & \multicolumn{2}{|c|}{ Grupo 2} \\
\hline & $\mathbf{n}$ & $\%$ & $\mathbf{n}$ & $\%$ \\
\hline \multicolumn{5}{|l|}{ Estado civil } \\
\hline Soltero & 188 & 81,4 & 178 & 75,4 \\
\hline Casado & 7 & 3,0 & 16 & 6,8 \\
\hline Separado/anulado & 10 & 4,3 & 15 & 6,4 \\
\hline Conviviente & 26 & 11,3 & 27 & 11,4 \\
\hline \multicolumn{5}{|l|}{ Vive con } \\
\hline Familia origen & 161 & 69,7 & 164 & 69,5 \\
\hline Familia nuclear & 20 & 8,7 & 29 & 12,3 \\
\hline Sólo pareja & 9 & 3,9 & 8 & 3,4 \\
\hline Otro & 24 & 10,4 & 26 & 11,0 \\
\hline Sólo & 7 & 3,0 & 9 & 3,8 \\
\hline Sin domicilio fijo & 10 & $4,3^{*}$ & 0 & $0,0^{*}$ \\
\hline \multicolumn{5}{|l|}{ Nivel educacional } \\
\hline Sin educación formal & 5 & $2,2^{*}$ & 0 & $0,0^{*}$ \\
\hline Básica completa/incompleta & 116 & $50,2^{*}$ & 48 & $20,3^{*}$ \\
\hline Media completa/incompleta & 106 & $45,9 *$ & 138 & $58,5^{*}$ \\
\hline Técnicos o universitarios & 4 & $1,7^{*}$ & 50 & $21,2^{*}$ \\
\hline Deserción escolar & 172 & $74,5^{* * *}$ & 92 & $39,0 * * *$ \\
\hline \multicolumn{5}{|l|}{ Situación laboral } \\
\hline Cesante & 107 & $46,3^{*}$ & 64 & $27,1^{*}$ \\
\hline Trabajo informal & 75 & $32,5^{*}$ & 45 & $19,1^{*}$ \\
\hline Trabajo formal & 26 & $11,3^{*}$ & 84 & $35,6^{*}$ \\
\hline Estudiante & 23 & $10,0^{*}$ & 43 & $18,2^{*}$ \\
\hline
\end{tabular}

${ }^{*} \chi^{2}$ (Residuos estandarizados); $\mathrm{p}<0,01 ;{ }^{* * *} \chi^{2}, \mathrm{P}<0,001$. 
Tabla 2. Comparación de patrones de consumo de último mes y de dependencia de sustancias entre consumidores de pasta base de cocaína (Grupo 1) y consumidores de clorhidrato de cocaína (Grupo 2) no consultantes de servicios de rehabilitación, al inicio, 6 meses y 12 meses de seguimiento

\begin{tabular}{|c|c|c|c|c|c|c|c|c|c|}
\hline \multirow[b]{2}{*}{ Variable } & \multicolumn{4}{|c|}{ Grupo 1} & \multicolumn{4}{|c|}{ Grupo 2} & \multirow[t]{2}{*}{ OR $(95 \%$ IC)* } \\
\hline & $\begin{array}{c}\text { B } \\
n\end{array}$ & $\begin{array}{l}\text { asal } \\
=231) \\
(\%)\end{array}$ & $\begin{array}{c}6 \mathrm{~ms} \\
(\mathrm{n}=190) \\
n(\%)\end{array}$ & $\begin{array}{c}12 \mathrm{~ms} \\
(\mathrm{n}=204) \\
n(\%)\end{array}$ & $\begin{array}{r}B \\
(n= \\
n\end{array}$ & $\begin{array}{l}\text { asal } \\
236) \\
(\%)\end{array}$ & $\begin{array}{c}6 \mathrm{~ms} \\
(\mathrm{n}=189) \\
n(\%)\end{array}$ & $\begin{array}{c}12 \mathrm{~ms} \\
(\mathrm{n}=198) \\
n(\%)\end{array}$ & \\
\hline \multicolumn{10}{|c|}{ Patrón de consumo } \\
\hline $\mathrm{PBC}^{\mathrm{a}}$ & 231 & (100) & $158(83,2)$ & $150(73,5)$ & 50 & $(21,2)$ & $34(18)$ & $39(19,7)$ & $28,02(19,2-40,8)$ \\
\hline $\mathrm{CC}^{\mathrm{b}}$ & 75 & $(32,5)$ & $53(27,9)$ & $54(26,5)$ & 236 & (100) & $144(76,2)$ & $131(66,2)$ & $0,08(0,06-0,12)$ \\
\hline Marihuana & 172 & $(74,5)$ & $121(63,7)$ & $111(54,4)$ & 173 & $(73,3)$ & $148(78,3)$ & $122(61,6)$ & $0,07(0,56-1,07)$ \\
\hline Anfetaminas & 12 & $(5,2)$ & $2(1,1)$ & $0 \quad(0)$ & 26 & (11) & $10(5,3)$ & $5(2,5)$ & $0,36 \quad(0,18-0,7)$ \\
\hline Solventes & 8 & $(3,5)$ & $2(1,1)$ & $4(2)$ & 4 & $(1,7)$ & $1 \quad(0,5)$ & $1 \quad(0,5)$ & $2,32(0,87-6,19)$ \\
\hline Sedantes & 26 & $(11,3)$ & $19(10)$ & $9(4,4)$ & 22 & $(9,3)$ & $15(7,9)$ & $10 \quad(5,1)$ & $1,22(0,75-1,97)$ \\
\hline Alcohol & 184 & $(79,7)$ & $155(81,6)$ & $149(73)$ & 213 & $(90,3)$ & $172(91)$ & $158(79,8)$ & $0,63(0,41-0,98)$ \\
\hline Policonsumo & 221 & $(95,7)$ & $164(86,3)$ & $159(77,9)$ & 230 & $(97,5)$ & $172(91)$ & $163(82,3)$ & $1,37(0,89-2,12)$ \\
\hline \multicolumn{10}{|l|}{ Dependencia $^{c}$} \\
\hline $\mathrm{PBC}^{\mathrm{a}}$ & 224 & $(97)$ & $146(92,4)$ & $141(94)$ & 39 & (78) & $32(94,1)$ & $38(97,4)$ & $2,35(1,16-4,79)$ \\
\hline $\mathrm{CC}^{\mathrm{b}}$ & 64 & $(85,3)$ & $37(71,2)$ & $40(74,1)$ & 217 & $(91,9)$ & $128(88,9)$ & $114(87)$ & $0,40(0,25-0,66)$ \\
\hline Marihuana & 145 & $(84,3)$ & $99(81,8)$ & $83(74,8)$ & 150 & $(86,7)$ & $119(80,4)$ & $81(66,4)$ & $1,08(0,74-1,59)$ \\
\hline Alcohol & 116 & $(63)$ & $92(59,4)$ & $78(52,3)$ & 124 & $(58,2)$ & $98(57)$ & $75(47,5)$ & $1,16(0,86-1,59)$ \\
\hline Polidependencia & 197 & $(86,8)$ & $119(67,2)$ & $115(63,5)$ & 189 & (84) & $128(76,6)$ & $105(67,3)$ & $1,16 \quad(0,84-1,6)$ \\
\hline
\end{tabular}

*Odds Ratios calculadas empleando Ecuaciones de Estimación Generalizada (1: grupo 1; 0: grupo 2). aPasta base de cocaína.

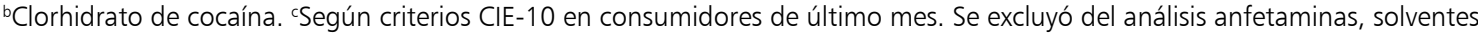
y sedantes debido al bajo número de casos.

\section{Análisis de retención}

La tasa de retención a los 12 meses fue $86,1 \%$ $(\mathrm{n}=402)$. La edad promedio de los 65 sujetos que abandonaron el estudio fue 22,7 años $(\mathrm{DE}=6,9)$, $y$ de los que completaron el seguimiento fue 23,7 años $(\mathrm{DE}=7,3)$, sin diferencia significativa entre ambos grupos $(t=1,022 ; \mathrm{p}=0,308)$. Hubo mayor retención de mujeres en el grupo $2(93,9 \%$ versus $\left.78,6 \% ; \chi^{2}[1,236]=9,3 ; \mathrm{p}=0,002\right)$. No se observaron diferencias por género en el grupo 1 .

En relación a las conductas de riesgo, sólo se observó una mayor proporción de negligencia en el cuidado de menores debido al consumo de drogas entre aquellos que abandonaron el estudio al interior del grupo 1 (grupo abandono: 69,2\% versus grupo retenido: $27,4 \% ; \chi^{2}[1,231]=8,6$; $\mathrm{p}=0,003)$.

\section{Patrón de consumo de sustancias}

En ambos grupos predominó el policonsumo, siendo la combinación más frecuente el tipo de cocaína que define el grupo (PBC o CC) asociada con alcohol y marihuana. En el grupo 1 se observó una proporción de sujetos que también consumía
CC, que osciló entre 32,5\% al inicio del estudio y $26,5 \%$ a los 12 meses. Igualmente, el grupo 2 evidenció un consumo de PBC en $21,2 \%$ de los sujetos al inicio y en 19,7\% al seguimiento. Existió una proporción significativamente mayor de consumidores de alcohol y anfetaminas en el grupo CC en relación al grupo PBC (Tabla 2). En todos los casos la vía de administración de PBC y marihuana fue la pulmonar, y nasal en caso de CC.

Respecto al diagnóstico de dependencia, no se observaron diferencias significativas en el consumo de marihuana y alcohol, siendo ambos grupos mayoritariamente dependientes de estas sustancias. Las únicas diferencias significativas correspondieron a las derivadas de los criterios de inclusión. Sin embargo, se observó un aumento en la proporción de adictos a la $\mathrm{PBC}$ durante el seguimiento en el grupo 2 (Tabla 2 ).

En relación a la severidad de la dependencia, los adictos a la PBC del grupo 1 presentaron una significativa mayor severidad de la adicción que los dependientes de PBC del grupo 2 (Inicio: 5,5 $[\mathrm{DE}=0,8]$ versus $4,5[\mathrm{DE}=1,1] ; 6$ meses: $5,4[\mathrm{DE}=$ $0,8]$ versus $5,1[\mathrm{DE}=0,9] ; 12$ meses: $5,4[\mathrm{DE}=0,9]$ 
Tabla 3. Comparación de conductas de riesgo entre consumidores de pasta base de cocaína (Grupo 1) y consumidores de clorhidrato de cocaína (Grupo 2) no consultantes de servicios de rehabilitación a los 6 y 12 meses de seguimiento

\begin{tabular}{|c|c|c|c|c|c|}
\hline \multirow[b]{2}{*}{ Conducta de Riesgo } & \multicolumn{2}{|c|}{ Grupo 1} & \multicolumn{2}{|c|}{ Grupo 2} & \multirow[t]{2}{*}{ OR $(95 \% \text { IC })^{*}$} \\
\hline & $\begin{array}{c}6 \mathrm{~ms} \\
(\mathrm{n}=190) \\
n(\%)\end{array}$ & $\begin{array}{c}12 \mathrm{~ms} \\
(\mathrm{n}=204) \\
n(\%)\end{array}$ & $\begin{array}{c}6 \mathrm{~ms} \\
(\mathrm{n}=189) \\
n(\%)\end{array}$ & $\begin{array}{c}12 \mathrm{~ms} \\
(\mathrm{n}=198) \\
n(\%)\end{array}$ & \\
\hline Sobredosis & $42(22,1)$ & $34(16,7)$ & $34(18)$ & $25(12,6)$ & $0,95(0,59-1,52)$ \\
\hline Conducta sexual de riesgo ${ }^{a}$ & $79(41,6)$ & $56(27,5)$ & $67(35,4)$ & $58(29,3)$ & $0,88(0,62-1,25)$ \\
\hline Haber abusado sexualmente de terceros & $4(2,1)$ & $5(2,5)$ & $5(2,6)$ & $3(1,5)$ & $1,07(0,41-2,81)$ \\
\hline Haber sido abusado sexualmente & $11(5,8)$ & $6(2,9)$ & $16(8,5)$ & $9(4,5)$ & $0,53(0,22-1,26)$ \\
\hline Consumo de drogas en relación sexual & $77(40,5)$ & $61(29,9)$ & $86(45,5)$ & $73(36,9)$ & $1,02(0,67-1,56)$ \\
\hline Heridas autoinferidas & $32(16,8)$ & $31(15,2)$ & $16(8,5)$ & $13(6,6)$ & $1,97(1,07-3,66)$ \\
\hline Intento de suicidio & $20(10,5)$ & $17(8,3)$ & $1(0,5)$ & $7 \quad(3,5)$ & $2,68(1,19-6,01)$ \\
\hline Trabajar bajo la influencia de sustancias & $53(27,9)$ & $56(27,5)$ & $68(36)$ & $54(27,3)$ & $1,05(0,65-1,70)$ \\
\hline Porte de armas ${ }^{b}$ & $97(51,1)$ & $89(43,6)$ & $78(41,3)$ & $64(32,3)$ & $1,29(0,88-1,88)$ \\
\hline Comisión de delitos ${ }^{c}$ & $86(45,3)$ & $81(39,7)$ & $59(31,2)$ & $59(29,8)$ & $1,13(0,78-1,67)$ \\
\hline
\end{tabular}

*Odds Ratios (OR) calculadas empleando Ecuaciones de Estimación Generalizada (1: grupo 1; 0: grupo 2). Las OR fueron ajustadas por potenciales factores confundentes: situación laboral, nivel educacional, deserción escolar, Disfunción familiar y síntomas emocionales (puntaje en Cuestionario de Salud General de Golberg-12). alncluyó: no usar anticoncepción, dar sexo para obtener

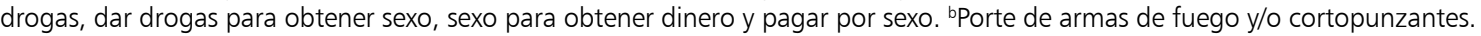
Incluyó: tráfico de drogas, robo en tiendas o supermercados, estafa, robo de autos o casas, asalto a personas y prostitución.

versus 5,3 [DE $=1]$; rANOVA: $\mathrm{F}=5,18 ; \mathrm{df}=2$; $\mathrm{p}<0,01$ en interacción tiempo-grupo). No se encontraron diferencias significativas al comparar la severidad de la dependencia a CC entre ambos grupos (Inicio: 4,7 [DE $=1,1]$ versus 5, $[\mathrm{DE}=1]$; 6 meses: $4,5[\mathrm{DE}=1]$ versus $4,9[\mathrm{DE}=1] ; 12$ meses: $4,8[\mathrm{DE}=1,2]$ versus $4,6[\mathrm{DE}=1,1]$; rANOVA: $\mathrm{F}=2,29 ; \mathrm{df}=2 ; \mathrm{p}=0,1$ en interacción tiempo-grupo) o marihuana (Inicio: $4,9[\mathrm{DE}=1]$ versus 4,8 $[\mathrm{DE}=1] ; 6$ meses: $4,7[\mathrm{DE}=1,1]$ versus $4,6[\mathrm{DE}=$ $1,2] ; 12$ meses: $4,4[\mathrm{DE}=1,1]$ versus $4,5[\mathrm{DE}=1,1]$; rANOVA: $\mathrm{F}=0,37 ; \mathrm{df}=2 ; \mathrm{p}=0,69$ en interacción tiempo-grupo) entre ambos grupos. La severidad de la dependencia a la PBC del grupo 1 fue significativamente mayor que la severidad de la dependencia a CC del grupo 2 (Inicio: $5,5[\mathrm{DE}=0,8]$ versus $5,1[\mathrm{DE}=1] ; 6$ meses: $5,4[\mathrm{DE}=0,8]$ versus $4,9[\mathrm{DE}=1] ; 12$ meses: $5,4[\mathrm{DE}=0,9]$ versus 4,6 $[\mathrm{DE}=1,1]$; rANOVA: $\mathrm{F}=2,91 ; \mathrm{df}=2 ; \mathrm{p}<0,05$ en interacción tiempo-grupo).

\section{Conductas de riesgo}

La Tabla 3 muestra que las conductas de riesgo más frecuentes en ambos grupos luego de
12 meses de seguimiento, fueron la comisión de delitos y porte de armas, seguido por conductas sexuales de riesgo y en tercer lugar sobredosis. Al seguimiento el grupo 1 mostró 2,7 veces mayor riesgo de intentar suicidio, y dos veces mayor riesgo de auto-inferirse heridas que el grupo 2. No se apreciaron diferencias significativas al comparar el resto de las conductas de riesgo.

\section{Discusión}

Los hallazgos principales luego de 12 meses de seguimiento indican que los consumidores de PBC tienen una mayor tasa de deserción escolar, desempleo y empleos informales que los consumidores de CC; respecto del patrón de consumo, ambos grupos fueron policonsumidores y polidependientes, predominantemente de cocaína (PBC o CC), marihuana y alcohol. La severidad de la dependencia a PBC fue significativamente mayor que la severidad de la dependencia a CC. Respecto de las conductas de riesgo, los consumidores de $\mathrm{PBC}$ presentaron una proporción significativa- 
mente mayor de heridas auto-inferidas e intentos de suicidio que los consumidores de CC.

Otro hallazgo relevante fue la alta tasa de retención a los 12 meses $(86,1 \%)$, que pudo haber sido determinado por la baja movilidad geográfica de los participantes y la tendencia a un funcionamiento adolescente que los hace dependientes de sus familias de origen ${ }^{14}$, entre otras posibles explicaciones.

La literatura señala que los consumidores que reciben tratamiento de rehabilitación reducen significativamente su patrón de consumo y las conductas de riesgo asociadas ${ }^{19-21}$, siendo el principal predictor de cambio la permanencia en tratamiento $^{22}$. En Chile sólo un estudio, efectuado por SENDA $^{23}$, ha evaluado el impacto del tratamiento en una cohorte de consumidores de drogas en el sistema público de rehabilitación, mostrando una significativa reducción en actos violentos en los 12 meses siguientes al término del tratamiento (53\% pre-tratamiento versus $15,1 \%$ post-tratamiento). Al comparar la evolución a los 12 meses de las conductas violentas de consumidores de cocaína no consultantes en nuestra muestra, podemos apreciar una reducción de 12,3\% en los consumidores de PBC y de 4,5\% en los consumidores de CC, cifras muy inferiores a 71,5\% de reducción de la cohorte que sí recibió tratamiento en el sistema público ${ }^{23}$.

Son múltiples los factores que determinan la mayor severidad de la dependencia y mayor prevalencia de autolesiones e intentos de suicidio en los consumidores de PBC en relación a los de CC. La vía de administración pulmonar se ha asociado a adicciones más severas ${ }^{24,25}$; la PBC contiene una variedad de sustancias tóxicas derivadas de los hidrocarburos ${ }^{2,26}$ que pueden tener algún rol en la aparición de las conductas de riesgo asociadas a la adicción. Otro factor reportado en los consumidores de PBC es la mayor disfuncionalidad familiar comparados con los consumidores de $\mathrm{CC}^{14}$. Crecer en familias poco protectoras puede aumentar el riesgo de consumo de sustancias ${ }^{27} y$ de adicción ${ }^{28}$. La deserción escolar aumenta cinco veces la probabilidad de consumo de sustancias ${ }^{16}$ y las bajas oportunidades laborales observadas en los consumidores de PBC pudiesen también determinar un mayor riesgo de consumo de drogas ${ }^{29}$.

Este estudio presenta algunas limitaciones: a) La selección de la muestra fue intencionada, lo cual fue minimizado mediante la estratificación por sexo y rango etario, el diseño de mapas de riesgo en cada comuna que delimitaron las zonas donde el consumo de drogas es mayor, y el reclutamiento de una muestra relativamente grande; b) La deseabilidad social de las respuestas, más aun, si los consumidores recibieron una compensación por participar en el seguimiento, pudiese haber distorsionado el reporte de las variables; c) El potencial efecto terapéutico de la interacción consumidor-entrevistador debe ser considerado. Aun cuando se hicieron esfuerzos por estandarizar y restringir los contactos con los consumidores, muy pocos entrevistadores interactuaron con los sujetos de estudio en momentos que no estaban programados en el diseño y no pudieron ser cuantificados; d) El análisis de las diferencias entre los sujetos retenidos y los que abandonaron reveló sólo tres diferencias significativas. En consecuencia, se consideró que la magnitud de la tasa de abandono no afectó de manera clínicamente relevante las diferencias observadas en las conductas de riesgo de los grupos de estudio; e) El CCR requiere de perfeccionamiento debido a la baja validez de ciertos constructos, específicamente sobredosis e intento de suicidio; f) Por razones presupuestarias, no se corroboró el consumo mediante análisis de sustancias en fluidos corporales.

A pesar de estas limitaciones se considera que los resultados obtenidos pueden ser extrapolables a los consumidores de PBC y CC de las comunas estudiadas que no han recibido tratamiento de rehabilitación, dados los múltiples resguardos a la validez y confiabilidad empleados en la recolección de información.

En conclusión, luego de un año de seguimiento, las conductas de riesgo más frecuentes de los consumidores de cocaína sin tratamiento son las conductas delictivas y sexuales de riesgo. Los consumidores de PBC presentan un perfil conductual de mayor riesgo vital que los consumidores de CC. Se requiere de estudios adicionales para explorar las relaciones causales de esta asociación, tales como el rol de enfermedades mentales subyacentes, la disfunción familiar o la marginalización social. La metodología empleada fue efectiva para contactar y seguir en el tiempo a consumidores de drogas en su medio natural. Si se considera la baja tasa anual de búsqueda de tratamiento ${ }^{7}$, es razonable recomendar la realización de estudios observacionales periódicos que permitan caracterizar clínicamente a consumidores no consultantes, con 
el objeto de diseñar intervenciones psicosociales precoces aplicables en el medio natural de los consumidores.

Agradecimientos: Agradecemos a FONDECYT por el financiamiento de esta investigación (Proyecto \#1030431).

\section{Referencias}

1. Jeri FR. Coca-paste smoking in some Latin American countries: a severe and unabated form of addiction. Bull Narc 1984; 36: 15-31.

2. El Sohly M, Brenneisen R, Jones AB. Coca paste: Chemical analysis and smoking experiments. J Forensic Sci 1991; 36: 93-103.

3. Montoya I, Chilcoat H. Epidemiology of coca derivatives use in the Andean region: a tale of five countries. Subst Use Misuse1996; 31: 1227-40.

4. Servicio Nacional para la Prevención y Rehabilitación del Consumo de Drogas y Alcohol. Séptimo Estudio Nacional de Drogas en Población General de Chile, 2006. Observatorio Chileno de Drogas, 2007. Disponible en: http://www.senda.gob.cl/wp-content/ uploads/2011/04/2006_septimo_estudio_nacional.pdf [Consultado el 21 de junio de 2015].

5. Pascale A, Hynes M, Cumsille F, Bares C. Consumo de pasta base de cocaína en América del Sur: Revisión de los aspectos epidemiológicos y médico-toxicológicos. OEA-CICAD. 2014. Disponible en: http://www.cicad. oas.org/oid/pubs/pbc.pdf [Consultado el 21 de junio de 2015].

6. Servicio Nacional para la Prevención y Rehabilitación del Consumo de Drogas y Alcohol. Décimo Primer Estudio Nacional de Drogas en Población General de Chile, 2014. Observatorio Chileno de Drogas, 2015. Disponible en: http://www.senda.gob.cl/wp-content/ uploads/2015/07/Resultados-principales-ENPG-20141. pdf [Consultado el 8 de julio de 2015].

7. United Nations Office on Drugs and Crime. World Drug Report 2014 (United Nations publication, Sales No. E.14.XI.7). Disponible en: http://www.unodc.org/ documents/wdr2014/World_Drug_Report_2014_web. pdf [Consultado el 21 de junio de 2015].

8. Wiebel W. Identifying and gaining access to hidden populations. NIDA Res Monogr 1990; 98: 4-11.

9. van de Goor LA, Garretsen HF, Kaplan C, Korf D, Spruit, IP, De Zwart WM. Research methods for illegal drug use in hidden populations: summary report of a European invited expert meeting. J Psychoactive Drugs 1994; 26: 33-40.
10. Griffiths P, Gossop M, Powis B, Strang J. Reaching hidden populations of drug users by privileged access interviewers: methodological and practical issues. Addiction 1993; 88: 1617-26.

11. Santis R, Hayden V, Ruiz S, Anselmo E, Torres R, Pérez De Los Cobos J. Implementación de la entrevista de acceso privilegiado para caracterizar usuarios de pasta base de cocaine. Rev Chil Neuro-Psiquiat 2004; 42: 273-80.

12. Santis R, Hayden V, Ruiz S, Anselmo E, Torres R, Hidalgo CG. Patrones de consumo de sustancias de una muestra no consultante de consumidores de pasta base de cocaine. Rev Chil Neuro-Psiquiat 2006; 44: 15-22.

13. Santis R, Hidalgo CG, Rodríguez J, Hayden V, Anselmo E. Consumo de sustancias y conductas de riesgo en consumidores de pasta base de cocaína y clorhidrato de cocaína no consultantes a servicios de rehabilitación. Rev Med Chile 2007; 135: 45-53.

14. Hidalgo CG, Santis R, Rodríguez J, Hayden V, Anselmo E. Family functioning of out-of-treatment cocaine base paste and cocaine hydrochloride users. Addict Behav 2008; 33: 866-79.

15. Jaramillo A, Hidalgo CG, Armijo I, Hayden V, Santis R, Lasagna A. Aspectos Clave en el Desempeño Exitoso de Agentes Comunitarios en Intervenciones de Outreach con Jóvenes Consumidores de Drogas. Psykhe 2014; 23 (1): 1-14.

16. Servicio Nacional para la Prevención y Rehabilitación del Consumo de Drogas y Alcohol. Cuarto Estudio Nacional de Drogas en Población General de Chile, 2000. Observatorio Chileno de Drogas, 2001. Disponible en: http://www.senda.gob.cl/wp-content/ uploads/2011/06/2000_Cuarto_EstudioNacional.pdf [Consultado el 21 de junio de 2015].

17. World Health Organization. The ICD-10 classification of mental and behavioural disorders: clinical descriptions and diagnostic guidelines. Geneva: WHO; 1992.

18. Araya R, Lewis G. Comparison of two self administered psychiatric questionnaires (GHQ-12 and SRQ-20) in primary care in Chile. Soc Psychiatry. Psychiatr Epidemiol 1992; 27: 168-73.

19. McLellan AT, Lewis DC, O’Brien CP, Kleber HD. Drug Dependence, a Chronic Medical Illness: Implications for Treatment, Insurance, and Outcomes Evaluation. JAMA 2000; 284: 1689-95.

20. Gossop M, Marsden J, Stewart D, Kidd T. The National Treatment Outcome Research Study (NTORS): 4-5 year follow-up results. Addiction 2003; 98: 291-303.

21. Hubbard R, Craddock G, Anderson J. Overview of 5-year followup outcomes in the drug abuse treatment outcome studies (DATOS). J Subst Abuse Treat 2003; 25: 125-34. 
22. Simpson DD. Modeling treatment process and outcomes. Addiction 2001; 96: 207-11.

23. Consejo Nacional para el Control de Estupefacientes. Seguimiento de una cohorte de beneficiarios para la evaluación de impacto de los planes de atención de las instituciones en convenio con CONACE-FONASA. 2007. Disponible en: http://www.bibliodrogas.gob.cl/ biblioteca/digitales/publico/TRATAMIENTO_CL_6404. pdf [Consultado el 21 de junio de 2015].

24. Jones R. The Pharmacology of Cocaine Smoking in Humans. NIDA Res Monogr 1990; 99: 30-41.

25. Strang J, Bearn J, Farrell M, Finch E, Gossop M, Griffiths $\mathrm{P}$, et al. Route of drug use and its implications for drug effect, risk of dependence and health consequences. Drug Alcohol Rev 1998; 17: 197-211.

26. Duffau B, Rojas S, Espinoza ME, Jofré S, Muñoz L. Estudio de la composición química de incautaciones de cocaína en Chile mediante HPTLC, GC/FID y FTIR.
Revista de Toxicología en Línea 2014; 42: 64-77. Disponible en: http://www.sertox.com.ar/modules.php?na$\mathrm{me}=$ Content $\& \mathrm{pa}=$ showpage $\& \mathrm{pid}=906$ [Consultado el 21 de junio de 2015].

27. Merikangas KR, Dierker L, Fenton B. Familial Factors and Substance Abuse: Implications for Prevention. NIDA Res Monogr 1998; 177: 12-41.

28. Cirillo S, Berrini R, Cambiaso G, Mazza R. Una Mirada a la literatura. En: Cirillo S, Berrini R, Cambiaso G, Mazza R, Editores. La familia del tóxico dependiente. Barcelona: Editorial Paidós; 1999. p. 21-45.

29. Servicio Nacional para la Prevención y Rehabilitación del Consumo de Drogas y Alcohol. Sexto Estudio Nacional de Drogas en Población General de Chile, 2004. Observatorio Chileno de Drogas, 2005. Disponible en: http://www.senda.gob.cl/wp-content/ uploads/2011/04/2004_sexto_estudio_nacional.pdf [Consultado el 21 de junio de 2015]. 